\title{
Metáfora y Teoría Informacional para la mejora del pensamiento formal en adolescentes con trastorno del neurodesarrollo intelectual leve
}

\author{
Paola VÁsquez EsPinoza \\ Universidad Nacional Mayor de San Marcos \\ paolincita1@gmail.com
}

"La única y real discapacidad de un ser humano está dentro, nunca fuera. Lo que se ve es pasajero, pero lo que no se logra detectar con una mirada es lo que determina la esencia de la existencia y aún eso lo restaura el Maestro si se lo permito"

(Serafín Contreras)

Para Eberth

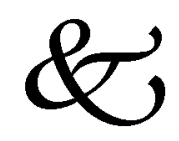

\begin{abstract}
Resumen
El diagnóstico que se enuncia para trabajar la mejora del pensamiento formal en personas que padecen el trastorno del desarrollo intelectual leve (TDI) (DSM-5 (317)), comúnmente conocido como síndrome de retraso mental leve (CIE-10 (F70)), es que el proceso de abstracción se encuentra seriamente dañado en ellas. Esta asociación está ligada a la preferencia en los postulados de factores biológicos, sin tomar en cuenta los componentes epigenéticos y sociocinéticos en contextos que únicamente observan la discapacidad conceptual como único indicador patológico y no consideran la interacción con el medio y otras directrices o factores que podrían intervenir. Si a ello se le une que los instrumentos a través de los cuales se mide y se diagnostica este tipo de alteración solamente se encuentran orientados a medir el coeficiente intelectual, se obtiene un espectro de posibilidades que coadyuvan a generar un clima de discriminación en países como el Perú y en otros con realidades semejantes.
\end{abstract}


Frente a este problema, se plantea la hipótesis de que los individuos que presentan la patología mencionada, sí son capaces de desarrollar la abstracción mediante la aplicación de un programa adecuado que se base en la metáfora cognitiva, por los lineamientos generales de la misma. En sujetos que no presentan el TDI, este tipo de aprendizaje resulta natural; sin embargo, para la población hacia la cual se pretende dirigir el estudio, la evidencia se encontraría ligada a la intervención, por parte del diseño, en situaciones que se incluyan en lo cotidiano y real. Por tal razón, se ha preferido que el trabajo esté dirigido a una muestra adolescente para evidenciar que el pensamiento formal -el cual empieza a determinarse desde inicios de la pubertad, a los 12 o 13 años, aproximadamente- también es posible de ser denotado, en su forma de abstracción, en individuos que presentan el TDI. El sistema a través del cual se orienta la diagramación de la presente exposición tiene como base el empleo de la metáfora cognitiva y la teoría informacional del Dr. Pedro Ortiz, a partir de un diseño descriptivo, el cual servirá para la experimentación posterior.

Palabras claves: Trastorno del desarrollo intelectual, metáfora cognitiva, pensamiento formal, doble determinación, Teoría informacional.

\section{Abstract}

The concept is stated to work improving the formal thinking in people who have been diagnosed with mild intellectual development (TDI) (DSM-5 (317)) disorder, commonly known as mild mental retardation syndrome (ICD-10 (F70)), is that the process of abstraction is seriously damaged them. This association is linked to the preference on the principles of biological, regardless social kinetis and epigenetic components observed in contexts that only the conceptual disabilities only pathological indicator and do not consider the interaction with the environment and other guidelines or factors that could intervene. If you are attached to it that the instruments through which is measured and this alteration only are oriented to measure IQ is diagnosed, a spectrum of possibilities that contribute to a climate of discrimination in countries is obtained as Peru and others with similar realities.

Faced with this problem, we hypothesized that individuals who have the disease mentioned, they are capable of developing abstraction by applying appropriate program is proposed that is based on cognitive metaphor, by the same general guidelines. In subjects who did not have the TDI, this type of learning is natural; however, for the population to which it is intended to conduct the study, the evidence would be found linked to the intervention, by design, to include situations in everyday life and real. For this reason, it is preferred that the work is aimed at 
an adolescent sample to show that formal thinking-which is initiated at since early adolescence, at 12 or 13 , approximately- denote it also be possible, as form of abstraction, in individuals with the TDI. The system through which the layout of this work is directed is based on the use of cognitive metaphor theory and informational Dr. Pedro Ortiz, from a descriptive design, which will be the basis for further experimentation.

Keywords: Intellectual development disorder, Cognitive metaphor, Formal thinking, Double determination, Information theory.

Recibido: 5/11/14 Aceptado: 28/11/14 Publicado on line: 10/2/15

\section{Introducción}

Durante muchos años, el trastorno del neurodesarrollo intelectual leve (TDI 317), inscrito como tal en la edición del DSM-5 (2013), como retraso mental (F70) en la CIE 10, versión revisada y que en la CIE-11 va a aparecer como trastorno del desarrollo intelectual en el año 2015, ha sido catalogado -desde la concepción psiquiátrica y psicológica- como un tipo de discapacidad, cuyo dominio conceptual, en las personas que la padecen, presenta una alteración considerable a nivel del pensamiento formal. Dentro de esta perspectiva, la abstracción es la forma más común de visualizar dicha patología, la cual va a ser representada con serias limitaciones.

Sin embargo, a pesar de la definición establecida y de acuerdo a los avances linguiísticos y neurológicos actuales, el objetivo de esta presentación es exponer, de manera teórica, que en la evaluación del pensamiento formal, mientras se siga incluyendo datos en los que solamente se adscriba comportamientos ligados a situaciones que no tomen en consideración aspectos epigenéticos del ser humano, no pueden ser instrumentos válidos en su totalidad para denotar la falta de abstracción mencionada. Además, explicar que el empleo de la metáfora, como instrumento de estructuración conceptual, a nivel del sistema cognitivo formal, resulta válido para poder determinar que el pensamiento no tiene límites y que aborda, en primer lugar el plano linguiístico, pero que trasciende dicha frontera y, por lo tanto, su aplicación es de carácter interdisciplinario y determinado en una sociedad.

Por tal razón, el trabajo en mención partirá de los postulados de la teoría informacional del Dr. Pedro Ortiz, la cual ubica a las Neurociencias en un enfoque social, así como su relación con la aplicación de la metáfora cognitiva en personas que tienen dañado el pensamiento abstracto, como son las que presentan el trastorno del desarrollo intelectual leve.

Esta situación resulta novedosa, ya que los trabajos existentes relacionados al estudio de la metáfora se ubican en diversas áreas; sin embargo, cuando se 
considera a los individuos con retraso mental leve como solía llamárseles, el hecho se inscribe en otra dirección. Se ha encontrado el estudio de Tamarit, de la confederación española de organizaciones a favor de las personas con discapacidad intelectual, quien explica la importancia de la sociedad y las familias en el desarrollo de los sujetos con TDI. Afirma que la situación no ha sido nada favorable con ellos en el transcurso de la historia del mundo; los postulados residen sobre todo en los problemas de trasfondo presentados, tales como la discriminación y la falta de oportunidades, por aspectos que se basan únicamente en el intelecto. En esta sección afirma que la metáfora, primero la aristotélica, luego la mecánica y actualmente la orgánica llevan a plantear dificultades a nivel de las diferencias. En su estudio considera que estas últimas sirven como proceso de asimetría entre unos y otros, más que de confluencia. (Tamarit, 2006)

Por su parte, Crespo Suárez de la Universidad Complutense de Madrid, en su artículo, plantea que las teorías en las ciencias humanas y sociales, se han construido siempre sobre el modelo metafórico del ser humano. Las que han prevalecido generalmente han sido las representacionistas y las computacionales. Desde esta perspectiva, tal como se ha desarrollado la vida y la construcción social, se hace inevitable la aparición de la metáfora en ella. Por tal razón, esta categoría sirve para generar nuevas opciones de acción en el contexto en el cual se presenten (Crespo Suárez, 2003).

A su vez, Fajardo Uribe de la Universidad Nacional de Colombia explica que la metáfora es un mecanismo de carácter cognitivo que hace posible la percepción del mundo a través de múltiples procesos que tienen que ver con "un dominio de origen y un dominio de llegada". La situación se inscribe como una necesidad básica de la persona para referirse a la realidad concreta, sin tener que crear nuevos conceptos y además se encuentra enriquecida con otros componentes tales como la afectividad y/o emotividad. (Fajardo, 2007)

Como se puede apreciar, los estudios se encuentran mucho más abocados a situaciones que consideran la aplicación de la metáfora a situaciones cotidianas o con los sujetos que no presentan ningún tipo de patología. Lo planteado en el presente documento es que la persona con trastorno del desarrollo intelectual leve puede llegar a obtener un nivel de abstracción si es que se aplica la práctica adecuada de un programa que se base en la metáfora cognitiva, ya que si bien es cierto dicha patología puede comportar una base genética y/o congénita, eso no la excluye de la intervención de procesos epigenéticos y sociocinéticos, que ayuden a mejorar la situación. Dicho enfoque será la base de esta exposición. 


\section{Trastorno del neurodesarrollo intelectual y la perspectiva social actual}

Desde la definición determinada por la Asociación Americana de Discapacidades Intelectuales y del Desarrollo, en su undécima edición (AAIDD- 2010) y que anteriormente fue llamada American Association Mental Retardation (AAMR) se concibe al trastorno del desarrollo intelectual a partir de cinco áreas específicas, que competen las dimensiones intelectuales; de conducta y adaptativas; de participación, interacción y roles sociales; de salud, y por último, el contexto. (Disabilities, 2010). Esta definición, que ya había sido planteada por la AAMR en el año 2002, presenta el cambio de paradigma en razón a que los que sufren la discapacidad intelectual no pueden ser definidos en el marco de un rasgo de carácter del individuo, sino como una interacción entre la persona y el contexto. A partir de esta conceptualización, la categoría diagnóstica establecida pasa a ser entendida como un estado de funcionamiento del ser. El otro hecho significativo aportado es el tema de los apoyos, puesto que el progreso del individuo radica en cuánta ayuda recibe del medio y la posibilidad del establecimiento de manera adecuada, a través de nueve áreas: desarrollo humano, enseñanza y educación, vida en el hogar, vida en la comunidad, empleo, salud y seguridad, manejo conductual, desarrollo social, y protección y defensa. Se propone, además, que todo ser humano puede progresar, ya que la deficiencia es un reto que se le impone al medio y que depende del entorno el proporcionar la mejora para el funcionamiento individual. Esta concepción está sustentada en un aspecto no determinista del fenómeno, sino que se la observa desde una concepción holística y que promueva la inclusión con base teórica en educación, salud pública, cognición y sociedad.

La situación fue planteada en razón al hecho de que en determinados contextos, las personas que padecían este trastorno eran (y son) catalogadas desde la perspectiva intelectual únicamente, olvidando algunos otros criterios que aparecen en el desarrollo integral del individuo. Además, si a ello se le aúna la caracterización que durante miles de años acompañó a las personas con TDI, la cual partió de una historia estereotipada y discriminadora, el cambio en cuanto al paradigma resulta novedoso y relevante, en razón al hecho que los límites no se encuentran marcados por conceptos que acompañen el postulado únicamente interno de la persona, sino que más bien se asocien a un conjunto de aplicaciones que correspondan al quehacer externo. Es interesante notar que desde la teoría piagetiana hasta nuestros días, la situación en razón al determinismo genético, y por tanto, implicado en una serie de conceptos al respecto de la patología que se está abordando, ha constituido el componente 
principal rescatado por los investigadores. Se ha dejado de soslayo aspectos que responden a criterios no deterministas, pero que no por ello dejan de tener sustento científico.

El resultado de la comprensión (no se habla de literalidad) es un hecho connatural y no solamente inferencial, ya que resulta de una práctica de la vida habitual en términos de abstracción y cognición en general. Por lo tanto, en ella está presente el conocimiento en general. No se puede negar el desarrollo de la cognición en personas que presentan TDI; por tal razón, la manifestación de la abstracción también se logra si se determinan a partir de una intervención adecuada en la cultura, que puede observarse en el aprendizaje general, el cual también debe ser el apropiado. Con ello, no se está negando la presencia de aspectos genéticos, solo que esta vez no se plantea desde una lectura puramente determinista, sino más bien como una relación que puede verse influencia por relaciones con el entorno inmediato.

\section{De lo epigénetico a lo sociocinético}

El concepto de epigénesis está configurado como la producción de una serie de cambios en el organismo a partir de factores no genéticos. A pesar que este concepto data del año 1942, en que Conrad Hall Waddington mencionó la interacción entre genes y ambientes (Celnikier, 2013), recién fue corroborado en el año 2003 con la finalización del Proyecto del Genoma Humano, que postula que aunque las personas vengan determinadas genéticamente por una serie de factores, estos pueden ser cambiados por las transformaciones que se hagan en el medio. Se trata de poder entender que la herencia juega un rol muy importante en el progreso del ser, pero que a lo largo de su existencia, desde el desarrollo celular, pasando por el tisular, orgánico, neural, inconsciente y consciente -en donde el nivel más alto corresponde al lenguaje- a partir de la teoría de Pedro Ortiz, se hace imperioso destacar la organización de datos procesados contenidos en cada uno de estos organismos (a manera de síntesis, véase el anexo 01). Es la información, en cualquiera de sus manifestaciones (genética, metabólica, neural, psíquica o social), la que va a permitir el desarrollo del individuo en cuanto a su relación con los demás.

Si se parte que actualmente, en el mundo, se está buscando la forma de descifrar la observación de cómo las propias experiencias pueden determinar el material genético (aunque esta situación hasta ahora resulte desconocida para los científicos), la ley de la doble determinación, propuesta por el Dr. Pedro Ortiz Cabanillas (1999, revisada en el 2004) aparece como totalmente válida. Dicha correspondencia incluye que la información social codificada, la cual se encuentra inscrita en el nivel consciente de la persona, puede visualizarse 
desde una perspectiva social y genética y ser influenciada mutuamente. Con esta relación de ida y vuelta no quedan dudas en cómo el medio influye en el progreso de una persona, aunque el problema haya venido condicionado con la herencia.

Por tal razón, se considera que el empleo de un programa, que busque su base en la metáfora, puede servir como un instrumento válido para poder estructurar pensamientos de carácter formal en personas con TDI. La justificación radicaría en los mecanismos cognitivos que se activan. El mundo que percibimos no solo se hace posible a través de la situación expresada en términos que empleen recursos únicamente linguiísticos, sino que también observamos que esa percepción puede escapar a los límites sensoriales. Por ejemplo, de acuerdo a estudios con neuroimagen desarrollados en la universidad de Emory (2012) se determinó que las áreas involucradas en la definición de textura que comprenden la percepción a partir del tacto se encuentran incluidas en el lóbulo parietal. Se puede establecer con toda confianza que el cerebro elabora metáforas, las cuales pueden ser determinadas como un tejido de un concepto que se encuentra contenido en otro, comportando las mismas características que denotan la actividad motora. Por ello, Glenys Álvarez menciona lo siguiente:

El lenguaje rebosa con metáforas. Es una forma de vida explicar las cosas con palabras distintas que representan un sentimiento, una forma de describir mucho mejor un pensamiento, una emoción, una expresión. No sólo la literatura, la academia y la prensa se valen de las metáforas para articular elegantemente, para enseñar, para divertir y entretener, sino que la cotidianidad navega en sus mares también, entregándose al símil, a las alegorías, la simbología y demás para dejar dicho en pocas palabras lo que sentimos [...]Ahora, nuevas investigaciones de imágenes en el cerebro indican que regiones importantes, como el opérculo parietal que reacciona a las texturas, se activan al escuchar una metáfora como "sus palabras ásperas me arañaban el alma". (Álvarez, 2012)

Por su parte, Krish Sathian, del centro de neurología, medicina de rehabilitación y psicología en Emory dice: "El resultado ilustra cómo extraemos entendimiento del lenguaje metafórico utilizando las experiencias de las sensaciones" (Álvarez, 2012).

Además, también en términos actuales, se puede expresar que de acuerdo a la estimulación que se desencadena en el medio, se activa un sistema de redes neurales con características diversas que difieren entre un individuo y otro. A este considerando se le denomina neuroplasticidad cerebral y el criterio que se establece como concepto para ella es que la activación de un elemento neural resulta de la interpretación que cada persona pueda hacer a partir de su experiencia, en donde intervienen factores psicológicos, sociales, innatos 
y demás. Por tanto, la novedad de este hecho es la capacidad que posee el cerebro para desarrollar y formar conexiones nerviosas, a través de los procesos sinápticos a lo largo de la toda la vida. Este concepto parte de la respuesta a los estímulos del medio y que pueden mejorar algún tipo de disfunción o daño. La mencionada actividad, que también es conocida y reconocida como "la renovación del cableado cerebral" es la que ha permitido encontrar diversas posturas, a partir de las cuales se observa que en un futuro cercano algunas enfermedades degenerativas y/o cognitivas podrán ser tratadas, tal como se aprecia en estudios realizados alrededor del mundo y como lo expresa la asociación Neurofeedback de Marbella:

El cerebro es un órgano extremadamente dinámico en permanente relación con el ambiente, por un lado, y con los hechos psíquicos o los actos del sujeto, por otro. Las últimas investigaciones de las neurociencias demuestran que el cerebro puede regenerarse mediante su uso o potenciación. (Cogny Legrand, 2011)

La pretensión de esta exposición es mostrar que a partir de un programa que oriente la educación de las personas con el trastorno del desarrollo intelectual, el cual se fundamente en la metáfora cognitiva, puede ser la fórmula para determinar aspectos de mejora inscritos en su esencia misma, ya que aunque se hablara de una situación de daño cerebral (que sería la forma extrema para considerar el TDI leve), incluso así se establecerían procesos cognitivos y linguísticos superiores, en los que intervienen los mecanismos propios de la metáfora y que devienen en la articulación con la neuroplasticidad del cerebro.

Los datos que se conocen se hacen factibles a partir de la interpretación que cada individuo pueda formar en una situación determinada; sin embargo, dichos componentes se estipulan en el ejercicio de una comunidad, en donde se conceptualiza una realidad, a partir de las ideas que se tengan sobre ella. Por lo tanto, la teoría de Ortiz nuevamente se hace presente

Cabe pues concluir en que, si bien nuestro cerebro es humano por su epigénesis, es realmente personal por su sociocinesis. Este cerebro personal debe ser pues explicado respecto de sus procesos internos en doble sentido, porque doble es su determinación: epigenética y cinética. (Ortiz Cabanillas, Cuadernos de Psicobiología Social. Introducción a una Psicobiología Social del hombre, 2009)

\section{¿Por qué el pensamiento formal?}

El modelo fisiológico de la adolescencia establece que esta es el resultado de una reacción en cadena, a través de la cual se regulan las modificaciones morfológicas periféricas de los receptores mediante la secreción hipotalámica 
que ocasiona la liberación de gonadotropinas que van desde la hipófisis a las diversas glándulas.

Desde el modelo sociocultural se determina que la adolescencia es un proceso que varía según la sociedad y el enfoque cultural que se le atribuya. En algunos casos se establece que no existe, tal como sucede en los habitantes de Samoa, y en otros, como sucede en el modelo de cultura occidentalizada peruana, es parte del seno de una familia.

Como se puede apreciar, la adolescencia constituye un fenómeno que varía de acuerdo al momento histórico y social. Lo que sí parece una consideración común es que involucra un cambio, el cual se encuentra determinado por la abstracción que conllevan una serie de procesos a través de los cuales las operaciones pasan del mundo real y concreto, de lo observable al mundo de lo posible e hipotético.

Para el caso de las personas con TDI se considera que esta capacidad se encuentra dañada, puesto que el desarrollo de las operaciones que permiten observar la metacognición y la eficacia que tenga que ver con lineamientos de carácter temporal, generalizaciones, síntesis, planteamiento argumentativo, algunos conceptos implicados no resulta evidenciable.

Dada la experiencia en el tratamiento de los individuos que presentan dicho trastorno, el trabajo actual plantea que la abstracción sí resulta posible, porque los conceptos que permanecen en la actuación de la vida cotidiana es lo que realmente va a permitir desarrollar lo que se encuentra "oculto" en el pensamiento de las personas en general y de las que tienen TDI, en particular. Se debe recordar que las personas con habilidades diferentes, como en muchas circunstancias se les llama, desarrollan otras áreas cognitivas, no descubiertas aún, pero no por ello inexistentes.

\section{Ahora sí la metáfora}

El concepto de metáfora fue desarrollado por Lakoff y Johnson en su libro Metaphors We Live By : "La metáfora impregna la vida cotidiana, no solamente el lenguaje, sino también el pensamiento y la acción”. (Lakoff, 2000- versión en español).

Vista desde esta circunstancia, la metáfora conlleva una situación real y totalmente necesaria en la vida del hombre:

La metáfora se nos presenta como un mecanismo que permite la conceptualización del mundo, la organización de este y la articulación de las concepciones que de él se tengan. Dado su dinamismo, es la única capaz de dar cuenta de la forma cómo percibimos ese constante cambio. La metáfora no solamente organiza o reorganiza la realidad que enfrentamos sino que es capaz de crearla y recrearla a partir de las conexio- 
nes que se establecen entre los elementos que la constituyen. (Fajardo, 2007)

Dicho concepto constituye un aspecto a través del cual las personas se comunican y son capaces de generar información que va a ser susceptible de codificarse en una realidad determinada. Frente a ello, se puede enunciar que si se trata de información que aparece en el mundo, entonces, el sistema cognitivo se activará de un modo consciente, por lo que será factible de emplearse en cualquier otro momento en situaciones semejantes. Frente a ello se puede afirmar:

Un sistema semiótico que tiene la propiedad de codificar los sistemas de signos creados por la sociedad, como es el lenguaje, en un sistema de memoria igualmente neocortical, que viene a ser el sistema del habla personal, en el nivel preconsciente del cerebro, es el que permite el desarrollo de la sociedad. (Ortiz Cabanillas, 2002)

El trabajo a nivel de la metáfora cognitiva constituye una apropiación que deviene en diversas ciencias, como la Linguiística, la Psicología, la Neurología, entre otras. Por tal razón, el concepto de lo denominado metafórico corresponde a la intervención de pensamientos que se encuentren relacionados con el contexto. Además, si a ello se le agrega la importancia que se traduce en términos del receptor, entonces se puede decir que la comprensión se logra a partir de un proceso de integración de elementos dispares, en donde se construye el significado y se expone en la situación experiencial del lenguaje. De igual forma, la realidad, concebida a partir de creencias y convenciones vigentes será condicional para poder expresar lo que se desea.

De acuerdo a lo planteado por Lakoff (Lakoff, 2000- versión en español), la metáfora constituye una situación que se apropia no solamente del componente linguiístico, sino -sobre todo- del componente comunicativo. En una sociedad en la que prima un tipo de lenguaje metafórico, el hablante debe apropiarse, además de lo linguiístico, ciertas circunstancias que evidencian asuntos culturales, por ejemplo.

La experiencia diaria conduce a la adquisición de metáforas primarias, que crean una fusión entre las experiencias subjetivas y los juicios con las experiencias sensomotoras. Casi todos nuestros conceptos abstractos se definen en términos de metáforas conceptuales. Además, permite proyectar patrones inferenciales de un dominio de origen a uno de destino y es el que hace posible teorizar sobre hechos científicos abstractos. La razón y las estructuras conceptuales son producto del funcionamiento de nuestros cuerpos, nuestros cerebros y su relación con el mundo. (Lakoff, 2000- versión en español).

Vista de esta manera la metáfora, se puede afirmar que el pensamiento no está orientado únicamente a partir del intelecto, sino también al funcionamiento 
de los detalles comunes que percibimos a través de los sentidos (la situación planteada a partir de la neuroimagen en Emory evidencia este hecho). En el mismo, texto, los autores sugieren que la metáfora no es accionar a través del cual las personas participan de una manera en la que se detienen a pensar para promover la expresión; muy por el contrario, se convierte en parte de la vida y está presente en cada uno de los actos del individuo de forma tal que su uso es absolutamente natural. Al resultar ser una condición que se considera manifiesta en el accionar del sujeto, entonces el presentarlo como un sistema conceptual que se usa para pensar y actuar, representado a través del mundo cotidiano, también resulta evidente. Por lo tanto, para los individuos que emplean el lenguaje y que padecen TDI, la circunstancia no los excluye de esta postura y, haciendo una analogía simple, la metáfora resultaría indispensable. $\mathrm{Al}$ respecto, en el texto se menciona:

Sobre la base de la evidencia linguística ante todo, hemos descubierto que la mayor parte de nuestro sistema conceptual ordinario es de naturaleza metafórica. Y hemos encontrado una forma de empezar a identificar detalladamente qué son exactamente las metáforas que estructuran la manera en que percibimos, pensamos y actuamos. (Lakoff, 2000- versión en español)

En realidad, no se trata solamente de expresar los diversos aspectos desde la perspectiva de la enunciación; sino, sobre todo, el poder idear dichos postulados desde la situación que considere el uso de la misma y que se determine a partir de un pensamiento formado. Por tal razón, las metáforas existen de esa manera, porque se encuentran en el sistema conceptual de la persona, en el nivel consciente de su producción y ayudan a desarrollar aspectos con los lineamientos establecidos en su determinación linguiística.

Por lo tanto, cuando se añade la circunstancia que presenta el modelo neurolinguí́stico de la metáfora y los fundamentos neurológicos que se encuentran implicados en ella, se puede establecer que los procesos se establecen para todos los individuos, puesto que la neuroplasticidad del cerebro permite expresar estos conceptos, incluidas las personas que padecen el trastorno del desarrollo intelectual leve. Generalmente, las características más comunes se establecieron en las áreas de Broca y Wernicke, que se ubican en la tercera circunvolución frontal y mitad posterior de la circunvolución temporal posterior, respectivamente, ambas inscritas en el hemisferio izquierdo, dominante para el desarrollo del lenguaje; sin embargo, actualmente se puede afirmar categóricamente que los procesos no solamente se aprecian a este nivel. Existen estudios que advierten que la metáfora estaría relacionada con la región del giro angular posterior.

La región del giro angular, que es mucho más grande en los homínidos que en otros primates, es decisiva para las metáforas y las abstracciones, 
dada su ubicación estratégica en el cruce de áreas especializadas en el procesamiento de señales del tacto, la audición y la visión. Y agrega: "Aunque es prematuro concluir que el giro angular es el "centro de las metáforas" del cerebro humano, sí parece probable que la evolución de esa región contribuyó enormemente a la evolución de habilidades de abstracción puramente humanas". (Ramachandran, 2007)

Además, el hemisferio derecho por su componente en el proceso mental que deviene en un aspecto determinado del proceso inferencial, es posible que también tenga parte en el empleo de la metáfora. La realidad es que los enunciados figurativos son parte del medio social y como tal activan un rango más amplio de información semántica, lo que permite aumentar la sensibilidad. Por tal razón, cabe mencionar que el hemisferio izquierdo estaría relacionado con un lenguaje proposicional, que involucraría un componente cognitivo; mientras que el hemisferio derecho plantearía la relación de un lenguaje emocional.

El desarrollo de la información se plantearía a partir de las redes neurales que se encuentran determinadas no únicamente genética, sino también epigenética y sociocinéticamente, lo que hace posible el procesamiento de la información social (Ortiz Cabanillas, Lenguaje y Habla personal, 2005). Se insiste, por tanto, en que la postura determinista planteada por los lineamientos al nivel de las personas con TDI leve no resulta válida, sino que esta va a ser determinada por el medio en el cual el uso de la metáfora esté claramente identificado y que va a constituir su propio desarrollo linguiístico, el cual permitirá el proceso de codificación y recodificación, planteando los diversos niveles de abstracción.

\section{Referencias bibliográficas}

AGUADO Díaz, A. L. (2001) Historia de las deficiencias. Madrid: Universidad Complutense de Madrid, Facultad de Psicobiopatología.

ÁLVAREZ, G. (2012) Las metáforas y sus caminos sensoriales en el cerebro.Emory: Universidad de Emory. Obtenido de http://www.hechosdehoy.com/las-metaforas-y-suscaminos-sensoriales-en-el cerebro-14753.htm

ARTIGAS PALLARÉS, J. (2011). Trastornos del neurodesarrollo. Barcelona: Sociedad de Neurología Pediátrica Española.

BOLÓ, H. (2014). El manifiesto Psiquiátrico. Obtenido de http://www.elmanifiesto.com/ articulos_imprimir.asp?idarticulo $=745$

BROTHWELL, D. (2014). Digging up Bones. Obtenido de Trapation, practice of boring: http://stephen-herero.smugmug.com/Photography/Photos-almost-used-insos/2897693_tLPSm3/156028836_kPVqXM9\#! $i=156028836 \& k=k P V q X M 9$

BURGUESS, C \& Ch. Chiarello. (2000) "Neurocognitive Mechanism underlying metaphor comprenhension and other figurativo languajes" en Metaphor and symbolic activity, 11(02). 
CELNIKIER, F. (2013) Epigenética. Obtenido de http://www.epigenetica.org/definiendo-laepigenetica-y-temas-relacionados/

COGNY LEGRAND, A. (2011). Neurofeedback. Obtenido de www.neurofeedbackmarbella. com/plasticidad.html

CRESPO SUÁREZ, E. (2003) "El construccionismo y la cognición social” en Política y Sociedad, 40 (02) Madrid: Universidad Complutense de Madrid;pp 15-26.

DISABILITIES, A. A. (2010). AAID. Obtenido de http://aaidd.org/us

FAJARDO URIBE, Luz (2006) "La metáfora como proceso cognitivo" en Forma y función, 19, Bogotá: Univesidad Nacional de Colombia; pp.47-56.

FAJARDO URIBE, Luz. (2011) Aproximación a los fundamentos neurológico de la metáfora. Colombia: Universidad Nacional de Colombia.

GÓMEZ CARDOSO, Á. L. (2008) “El retraso mental. Antecedentes y perspectiva” en Estrategia educativa para la preparación de la familia del niño y la niña con diagnóstico de retraso mental, La Habana-Cuba: Camagiiey; pp. 65-66.

LAKOFF, G. y Mark JOHNSON (2001) Metáforas de la vida cotidiana. Madrid, Cátedra.

MARÍN ARRESE, J. I. (2011) Estudio contrastivo de la metáfoa en el discurso periodístico. Madrid: Tesis doctoral de la Universidad Complutense de Madrid, Facultad de Filología, Departamento de Filología Inglesa I.

ORTIZ CABANILLAS, Pedro (2002) Lenguaje y Habla Personal. Lima: Fondo Editorial San Marcos.

ORTIZ CABANILLAS, Pedro (2005) Lenguaje y habla personal. Lima- segunda edición: Fondo Editorial San Marcos.

ORTIZ CABANILLAS, Pedro (2009) Cuadernos de Psicobiología Social. Introducción a una Psicobiología Social del hombre. Lima: Fondo Editorial San Marcos.

ORTIZ DE ZÁRATE, Amaya (2000). La metáfora y la incertidumbre informacional. Madrid: Tesis doctoral de la Universidad Complutense de Madrid, Facultad de Psicología.

PARENTE, D. (2009) Literalidad, metáfora y cognición. La Plata: Universidad de La Plata. Obtenido de http://serbal.pntic.mec.es/ cmunoz11/dimeta2.pdf

RAMACHANDRAN, V. (2007). The Tell-Tale Brain. California: Center for Brain and Cognition.

SCHEERENBERGER, R. (1984). A history mental retardation. Baltimore: Brooks Producing .

TAMARIT, J. (2006) “Discapacidad intelectual” en G. \&. Millas Manual de Atención Temprana. Valencia:Promolibro; pp. 663-682. 
